\title{
Magnon Transport in Quasi-Two-Dimensional van der Waals Antiferromagnets
}

\author{
Wenyu Xing, ${ }^{1,2}$ Luyi Qiu, ${ }^{1,2}$ Xirui Wang, ${ }^{1,2}$ Yunyan Yao, ${ }^{1,2}$ Yang Ma, ${ }^{1,2}$ \\ Ranran Cai, ${ }^{1,2}$ Shuang Jia, ${ }^{1,2,3}$ X. C. Xie, ${ }^{1,2,3}$ and Wei Han ${ }^{1,2 *}$ \\ ${ }^{1}$ International Center for Quantum Materials, School of Physics, Peking University, \\ Beijing 100871, Peoples Republic of China \\ ${ }^{2}$ Collaborative Innovation Center of Quantum Matter, Beijing 100871, Peoples Republic of China \\ ${ }^{3}$ CAS Center for Excellence in Topological Quantum Computation, \\ University of Chinese Academy of Sciences, Beijing 100190, Peoples Republic of China
}

(Received 22 August 2018; revised manuscript received 12 December 2018; published 7 February 2019)

\begin{abstract}
The recent emergence of 2D van der Waals magnets down to atomic-layer thickness provides an exciting platform for exploring quantum magnetism and spintronics applications. The van der Waals nature stabilizes the long-range ferromagnetic order as a result of magnetic anisotropy. Furthermore, giant tunneling magnetoresistance and electrical control of magnetism have been reported. However, the potential of 2D van der Waals magnets for magnonics, magnon-based spintronics, has not been explored yet. Here, we report the experimental observation of long-distance magnon transport in quasi-twodimensional van der Waals antiferromagnet $\mathrm{MnPS}_{3}$, which demonstrates the 2D magnets as promising material candidates for magnonics. As the $2 \mathrm{D} \mathrm{MnPS}_{3}$ thickness decreases, a shorter magnon diffusion length is observed, which could be attributed to the surface-impurity-induced magnon scattering. Our results could pave the way for exploring quantum magnonics phenomena and designing future magnonics devices based on 2D van der Waals magnets.
\end{abstract}

DOI: 10.1103/PhysRevX.9.011026

Subject Areas: Condensed Matter Physics, Materials Science, Spintronics

\section{INTRODUCTION}

The recent emergence of two-dimensional (2D) van der Waals magnets down to atomic-layer thickness has attracted considerable interest and provided an exciting platform for exploring new physical phenomena in lowdimensional magnetism [1-15]. The long-range ferromagnetic order in 2D magnets has been demonstrated in bilayer $\mathrm{Cr}_{2} \mathrm{Ge}_{2} \mathrm{Te}_{6}$ and single-layer $\mathrm{CrI}_{3}$ as a result of magnetic anisotropy $[1,2,16]$. Shortly, the potential of such van der Waals magnets for spintronics applications has been intensively explored. For example, giant tunneling magnetoresistance in bilayer $\mathrm{CrI}_{3}$ has been demonstrated [17-19], which is much higher compared to conventional single-crystalline- $\mathrm{MgO}$ barrier-based ferromagnetic tunneling junctions $[20,21]$. The important role of magnonassistant tunneling through thin $\mathrm{CrBr}_{3}$ barriers has been shown in the graphene $/ \mathrm{CrBr}_{3} /$ graphene heterostructures [22]. Because of their 2D nature, efficient electrical control of magnetism in 2D ferromagnetic materials has also been

*weihan@pku.edu.cn

Published by the American Physical Society under the terms of the Creative Commons Attribution 4.0 International license. Further distribution of this work must maintain attribution to the author(s) and the published article's title, journal citation, and DOI. explored [3,23-26], which provides an alternative route towards high-temperature ferromagnetic semiconductors $[27,28]$. Furthermore, room-temperature 2D ferromagnetism in monolayer van der Waals magnet has also been demonstrated in epitaxial films and ionic liquid gated flakes $[8,9,25]$.

Magnonics refers to the magnon-based spintronics, the use of magnon-mediated spin current for information logic and computing applications [29]. One of the major research directions is to search the suitable magnon transport channel materials, which can propagate magnons over a long distance. Recently, the long-distance magnon transport has been demonstrated in 3D ferromagnetic and antiferromagnetic insulators, such as $\mathrm{YIG}, \mathrm{Cr}_{2} \mathrm{O}_{3}$, and $\mathrm{Fe}_{2} \mathrm{O}_{3}$ [30-32]. Specially, antiferromagnetic $\mathrm{Fe}_{2} \mathrm{O}_{3}$ offers an electrically tunable magnon channel that could be used for magnonics devices that could operate in the absence of any magnetic field [32]. Different from 3D magnetic insulators, such 2D van der Waals magnets offer special platforms for very intriguing quantum transport phenomena, including the spin Nernst effect, topological and Weyl magnons that have already been intensively studied theoretically [33-35]. However, the potential of 2D van der Waals magnets for magnon transport has not been experimentally investigated yet.

Here, we report the magnon transport properties in 2D van der Waals antiferromagnet $\mathrm{MnPS}_{3}$. The long-distance 
magnon propagation over several micrometers in $2 \mathrm{D}$ $\mathrm{MnPS}_{3}$ has been demonstrated, which is comparable to the 3D ferromagnetic insulator yttrium iron garnet (YIG). Systematical studies on the spacing dependence of the nonlocal magnon signal reveal a magnon relaxation length of several micrometers. As the temperature decreases, a longer magnon relaxation length is observed, which could be related to longer magnon lifetimes in such a van der Waals antiferromagnet at lower temperatures. As the 2D $\mathrm{MnPS}_{3}$ thickness decreases, a shorter magnon diffusion length is observed, which could be attributed to the surfaceimpurity-induced magnon scattering. These experimental results have demonstrated $2 \mathrm{D}$ van der Waals magnets as a new platform for the magnonics applications [29,36], and could further pave the way for exploring magnon-dependent quantum transport phenomena in 2D van der Waals magnets [33-35,37].

\section{EXPERIMENTAL}

Figure 1(a) illustrates the magnon transport in the quasi2D van der Waals antiferromagnet $\mathrm{MnPS}_{3}$ devices, where the $2 \mathrm{D} \mathrm{MnPS}_{3}$ flakes are prepared on the $\sim 300 \mathrm{~nm} \mathrm{SiO}_{2} / \mathrm{Si}$ substrates from bulk $\mathrm{MnPS}_{3}$ single crystals using the mechanical exfoliation method [23]. Bulk $\mathrm{MnPS}_{3}$ single crystals are grown using the chemical vapor transport method. Stoichiometric amounts of high-purity manganese, red phosphorus, and sulfur were sealed into an evacuated quartz tube in a temperature gradient from $780{ }^{\circ} \mathrm{C}$ (source region) to $730^{\circ} \mathrm{C}$ (growth region) for seven days. Figure 1(b) shows the crystalline and spin structures of the van der Waals antiferromagnet $\mathrm{MnPS}_{3}$. In each layer of the crystal's $a b$ plane, the spins of the Mn atoms are antiferromagnetic coupled with their nearest neighbors, while the interlayer exchange coupling between the $\mathrm{Mn}$ spins is ferromagnetic [38]. The Néel temperature $\left(T_{N}\right)$ of $\mathrm{MnPS}_{3}$ bulk single crystals is $\sim 79 \mathrm{~K}$, obtained from temperature-dependent magnetization measurement (Supplemental Material, Fig. S1 [39]) under the magnetic fields parallel and perpendicular to the crystal's $a b$ plane in a Magnetic Properties Measurement System (MPMS; Quantum Design).

The prepared 2D MnPS 3 flakes on $\sim 300 \mathrm{~nm} \mathrm{SiO}_{2} / \mathrm{Si}$ substrates are first identified by a Nikon high-resolution optical microscope, and then fabricated for the nonlocal magnon devices using standard electron-beam lithography and lift-off processes. The electrodes are made of 10-nmthick Pt grown in a magneton sputtering system with a base pressure lower than $8 \times 10^{-7}$ mbar. The width of the $\mathrm{Pt}$ electrodes is $\sim 200 \mathrm{~nm}$. Figure 1(c) shows the optical image of a typical magnon device made on 8-nm 2D MnPS 3 flake, where the thickness is determined by atomic force microscopy (Supplemental Material, Fig. S2 [39]). Raman studies show that the electron-beam lithography and device fabrication processes are not damaging the $\mathrm{MnPS}_{3}$ flakes (Supplemental Material, Fig. S3 [39]).

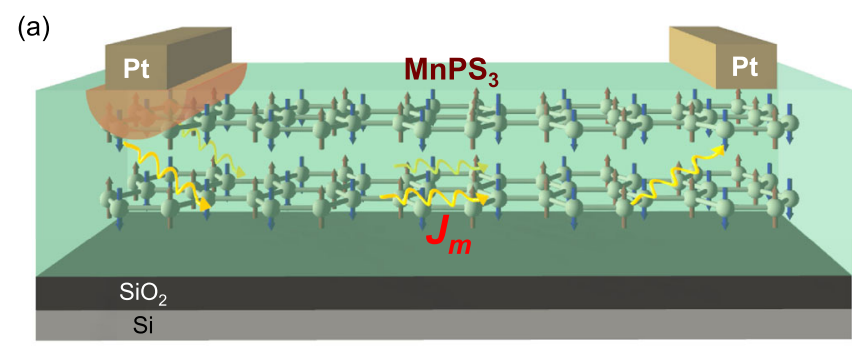

(b)

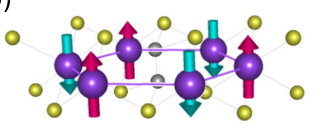

(c)
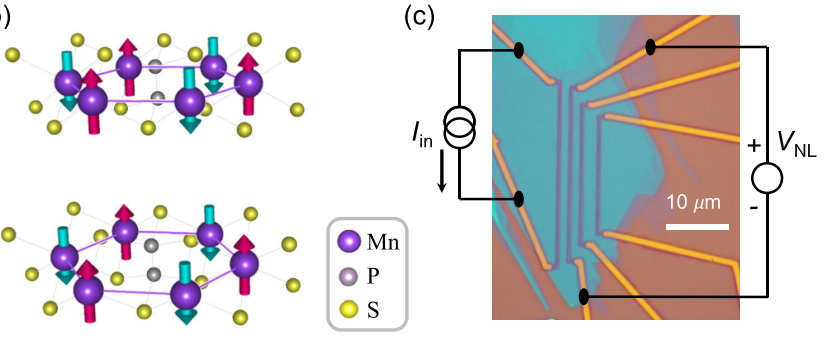

FIG. 1. Illustration of magnon transport in quasi-2D antiferromagnet $\mathrm{MnPS}_{3}$ devices. (a) Schematic of magnon transport in the quasi-2D $\mathrm{MnPS}_{3} . \boldsymbol{J}_{\boldsymbol{m}}$ represents the magnon-mediated spin current. The left Pt electrode is used as magnon injector via thermal spin injection, and the right $\mathrm{Pt}$ electrode is used as magnon detector via inverse spin Hall effect. (b) The crystal and spin structures of van der Waals antiferromagnet $\mathrm{MnPS}_{3}$. (c) Schematic of nonlocal measurement on a typical $\mathrm{MnPS}_{3}$ device via the low-frequency lock-in technique. The thickness of the $\mathrm{MnPS}_{3}$ flake is $8 \mathrm{~nm}$ determined via atomic force microscopy (Supplemental Material, Fig. S2 [39]).

The magnon transport in the quasi-2D van der Waals antiferromagnet $\mathrm{MnPS}_{3}$ is measured using the nonlocal geometry via standard low-frequency lock-in technique in a Physical Properties Measurement System (PPMS; Quantum Design). During the nonlocal magnon transport measurement, a current source (Keithley K6221) is used to provide the low-frequency ac current $(f=7 \mathrm{~Hz})$ in the range from 10 to $150 \mu \mathrm{A}$ in the spin injector Pt electrode, and the nonlocal voltages are measured using lock-in amplifiers (Stanford Research SR830). The voltage probes the magnon-dependent chemical potential due to magnon diffusion in the quasi-2D $\mathrm{MnPS}_{3}$ channel. During the measurement, low noise voltage preamplifiers (Stanford Research SR560) are used to enhance the signal-to-noise ratio.

The magnons are generated in quasi-2D van der Waals antiferromagnet $\mathrm{MnPS}_{3}$ under the left Pt electrode (magnon injector), and then diffuse towards the right Pt electrode (magnon detector) which detects the magnon-mediated spin current via inverse spin Hall effect of $\mathrm{Pt}$ in the nonlocal geometry [30-32,40,41]. To perform magnon injection, both electrical means via spin Hall effect of $\mathrm{Pt}$ and thermal means via thermal spin injection could be utilized [30], which give rise to the first and second harmonic nonlocal voltages probed at the magnon detector ( $V_{1 \omega}$ and $V_{2 \omega}$ ), respectively. Both means could be used to investigate the magnon transport properties in magnetic 
insulators, as demonstrated in previous reports [41-44]. In our experiment with the ac injection current $\left[I_{\text {in }}\right.$ in Fig. 1(c)] in the range from 10 to $150 \mu \mathrm{A}$, only the second harmonic nonlocal voltages could be clearly observed, while no obvious first harmonic voltages could be detected (Supplemental Material, Fig. S4 [39]). This result could be attributed to higher-efficiency magnon generation via thermal heating than that via the electrical means due to spin Hall effect of Pt. Hence, to probe the magnon transport in quasi-2D van der Waals $\mathrm{MnPS}_{3}$, thermal means is utilized to generate the magnons arising from the temperature gradient at the $\mathrm{MnPS}_{3}$-Pt interface via Joule heating $[30,31,42,45]$.

\section{RESULTS AND DISCUSSION}

Figure 2(a) shows the nonlocal magnon transport and measurement geometry in $\mathrm{MnPS}_{3}$, and Fig. 2(b) shows the second harmonic resistance $\left(R_{2 \omega}=\sqrt{2} V_{2 \omega} / I_{\text {in }}^{2}\right)$ as a function of the magnetic field angle $(\varphi)$ measured on the nonlocal magnon devices of 8-nm (black) and 16-nm (blue) $\mathrm{MnPS}_{3}$ flakes, with spacing $(d)$ of $2 \mu \mathrm{m}$ for both devices measured at $T=2 \mathrm{~K}$. During the measurement, the (a)

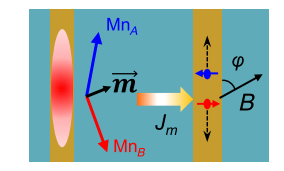

(c)

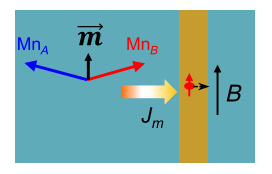

(d)

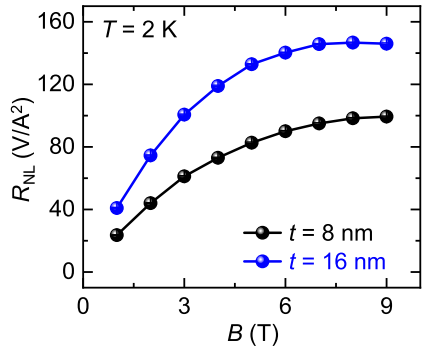

(b)

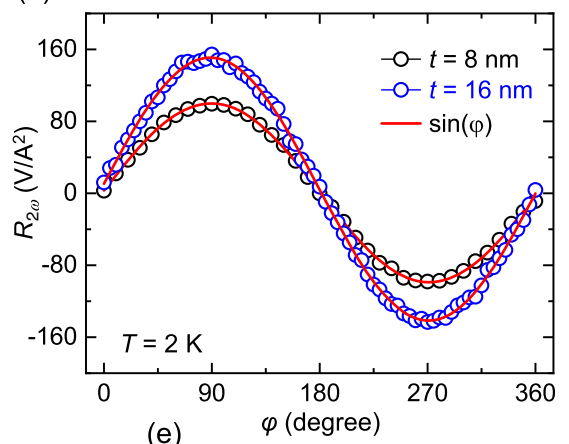

(e)

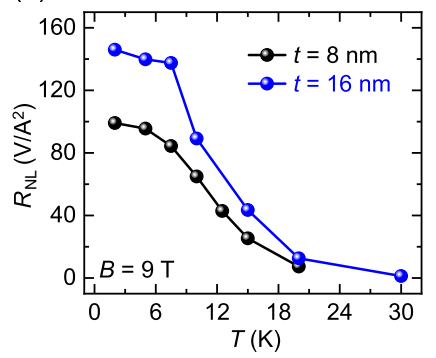

FIG. 2. Nonlocal magnon transport measurements in quasi-2D antiferromagnet $\mathrm{MnPS}_{3}$. (a) Schematic of the generation, transport, and detection of magnon-mediated spin current in $\mathrm{MnPS}_{3}$ magnon devices. (b) The nonlocal resistances of the 8-nm and 16-nm $\mathrm{MnPS}_{3}$ devices $(d=2 \mu \mathrm{m})$ as a function of the in-plane magnetic field angle at $T=2 \mathrm{~K}$ and $B=9 \mathrm{~T}$. (c) Illustration of the zero voltage signal when the magnetic field angle is $0^{\circ}$. (d) The in-plane magnetic field dependence of the nonlocal magnon signal for both $\mathrm{MnPS}_{3}$ devices at $T=2 \mathrm{~K}$. (e) The temperature dependence of the nonlocal magnon signal for both $\mathrm{MnPS}_{3}$ devices at $B=9 \mathrm{~T}$. in-plane static magnetic field $(B)$ is held at $9 \mathrm{~T}$, which gives rise to a canted magnetic moment $(\vec{m})$ between $\mathrm{Mn}_{A}$ and $\mathrm{Mn}_{B}$ in different magnetic sublattices [Fig. 2(a)]. The magnon-mediated spin current $\left(J_{m}\right)$ carrying the angular momentum parallel to $\vec{m}$ will be converted to the electronmediated spin current in Pt. Thus, the spin accumulation direction is parallel to $\vec{m}$. When the magnetic field angle is $90^{\circ}\left(-90^{\circ}\right)$, the maximum (minimum) voltages will be detected. On the other hand, if the magnetic field angle is $0^{\circ}$, as illustrated in Fig. 2(c), zero voltage signal is detected via inverse spin Hall effect since the spin accumulation direction is parallel to the Pt electrode. The 2D $\mathrm{MnPS}_{3}$ magnon devices are rotated from $0^{\circ}$ to $360^{\circ}$ in an in-plane static magnetic field, leading to the $2 \pi$-periodic rotation of $\vec{m}$. No clear signals of first harmonic nonlocal voltage could be detected under the ac injection current from 10 to $150 \mu \mathrm{A}$, which could be attributed to lower efficiency of magnon generation via the electrical means compared to the thermal means (Supplemental Material, Fig. S4 [39]). This observation is in accordance with previous reports on ferromagnetic insulator YIG and antiferromagnetic insulator $\mathrm{Cr}_{2} \mathrm{O}_{3}[30,31,45]$. Consistent with expectations for the transport of incoherent magnons [30,31], the second harmonic nonlocal voltage scales quadratically with the injection current (Supplemental Material, Fig. S5 [39]).

Since the 2D $\mathrm{MnPS}_{3}$ is insulating (Supplemental Material, Figs. S6 and S7 [39]), the flow of any charge current in the $2 \mathrm{D} \mathrm{MnPS}_{3}$ is forbidden, which rules out any nonlocal voltage arising from charge current leakage in the $\mathrm{MnPS}_{3}$ channel. Furthermore, the absence of the $R_{2 \omega}$ signal in the control samples fabricated directly on the $\mathrm{SiO}_{2} / \mathrm{Si}$ substrates confirms that the $R_{2 \omega}$ signal can only be obtained on the $\mathrm{MnPS}_{3}$ devices via magnon transport (Supplemental Material, Fig. S8 [39]).

As discussed earlier, since the magnons are injected via thermal means and the diffusive magnons are detected via the inverse spin Hall effect of Pt, $R_{2 \omega}$ is expected to be proportional to $\sin (\varphi)[30]$ :

$$
R_{2 \omega}=R_{\mathrm{NL}} \sin (\varphi),
$$

where the $R_{\mathrm{NL}}$ is the nonlocal spin signal. The red solid lines in Fig. 2(b) are best-fitting curves based on Eq. (1), from which $R_{\mathrm{NL}}$ is determined to be $99 \mathrm{~V} / \mathrm{A}^{2}$ for the $8-\mathrm{nm}$ $\mathrm{MnPS}_{3}$ device and $146 \mathrm{~V} / \mathrm{A}^{2}$ for the 16-nm MnPS 3 device, respectively. Obviously, a larger nonlocal magnon signal $\left(R_{\mathrm{NL}}\right)$ is observed for 16-nm $\mathrm{MnPS}_{3}$ device, which could be attributed to longer magnon relaxation lengths for thicker $\mathrm{MnPS}_{3}$ and be discussed later in detail. The in-plane magnetic field dependence of the nonlocal magnon signals on both devices is shown in Fig. 2(d) $(T=2 \mathrm{~K})$. As inplane magnetic field increases, the canted magnetic moment increases, giving rise to the enhancement of the second harmonic nonlocal magnon signals [31]. Figure 2(e) shows the temperature dependence of the nonlocal magnon 
(a)

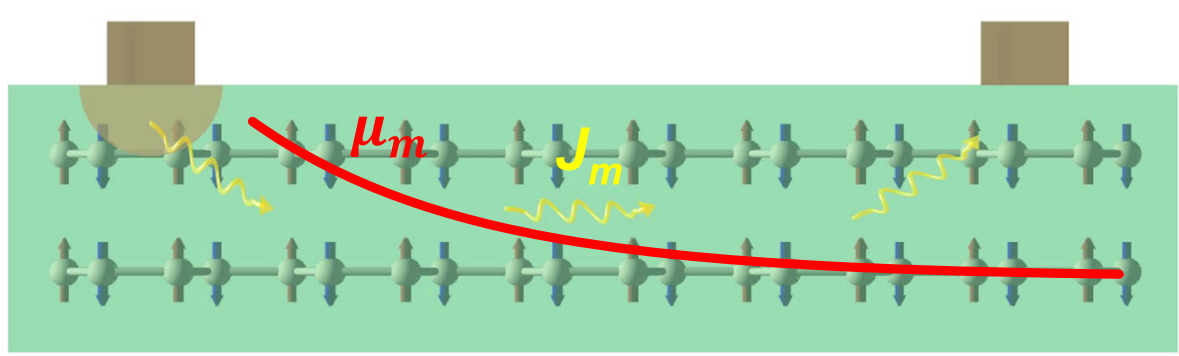

(b)
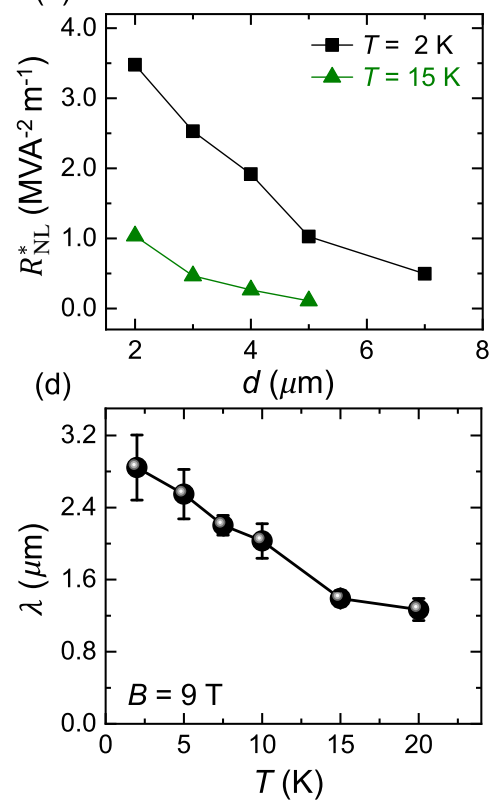

(c)

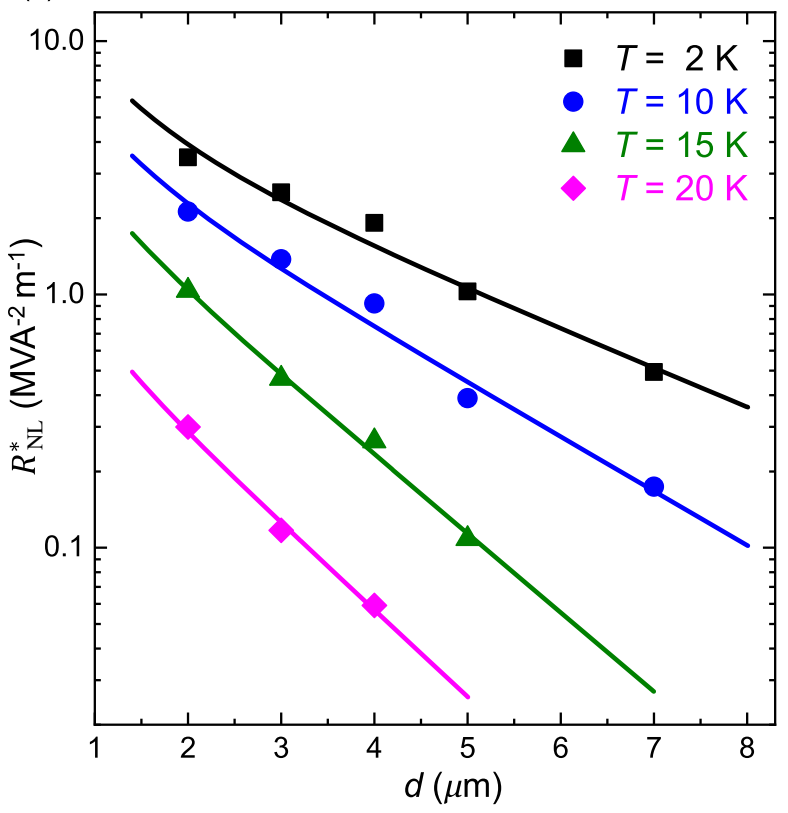

FIG. 3. The measurement of magnonic properties in quasi-2D antiferromagnet $\mathrm{MnPS}_{3}$. (a) Schematic of the exponential decay (solid red line) of magnon-dependent chemical potential $\left(\mu_{m}\right)$ during the flow of magnon-mediated spin current $\left(J_{m}\right)$. For simplicity, the magnon depletion below the Pt injector is not shown here (see Supplemental Material, Fig. S9 for details). (b) The spacing (d) dependence of the normalized nonlocal spin signals $\left(R_{\mathrm{NL}}^{*}\right)$ of the 16-nm MnPS $\mathrm{Mevice}_{3}$ measured at $T=2$ and $15 \mathrm{~K}$. (c) The spacing $(d)$ dependence of the normalized nonlocal spin signals $\left(R_{\mathrm{NL}}^{*}\right)$. The solid lines represent the best-fitting results based on Eq. (3). (d) The temperature dependence of the magnon relaxation length for the 16-nm $\mathrm{MnPS}_{3}$ device.

signals of both devices at $B=9 \mathrm{~T}$. The nonlocal magnon signals are observed when the temperature is lower than $\sim 20 \mathrm{~K}$ for the 8-nm $\mathrm{MnPS}_{3}$ device and $\sim 30 \mathrm{~K}$ for the 16$\mathrm{nm} \mathrm{MnPS}$ device, which could be attributed to lower Néel temperature for thinner $\mathrm{MnPS}_{3}$, a Heisenberg antiferromagnet [46]. These results demonstrate the potential of using a quasi-2D van der Waals antiferromagnet for the magnon transport, which might have advantages compared to ferromagnetic insulator YIG, such as the capability of functioning in the presence of large magnetic fields and the absence of stray fields $[47,48]$.

To investigate the magnon transport properties of quasi-2D van der Waals antiferromagnet $\mathrm{MnPS}_{3}$, the spacing profile of the magnon-dependent chemical potential $\left(\mu_{m}\right)$ is systematically studied. Since the $\mathrm{MnPS}_{3}$ thickness is much smaller than the spacing between the two $\mathrm{Pt}$ electrodes, magnon transport is expected to follow the one-dimensional drift-diffusion model $[30,49]$. As illustrated in Fig. 3(a), $\mu_{m}$ is expected to exponentially decay as the magnon-mediated spin current diffuses away from the magnon injector in the presence of magnon scatterings. At the distance of $d$ away from the magnon injector, $\mu_{m}$ can be described by the following expression:

$$
\mu_{m}=\frac{\mu_{0}}{\lambda} \frac{\exp \left(\frac{d}{\lambda}\right)}{1-\exp \left(\frac{2 d}{\lambda}\right)},
$$

where $\mu_{0}$ is the magnon-dependent chemical potential in $\mathrm{MnPS}_{3}$ under the Pt injector and $\lambda$ is the magnon relaxation length. Quantatively, the decrease of the nonlocal magnon resistances as a function of $d$ can be expressed by $[41,42]$

$$
R_{\mathrm{NL}}=\frac{C}{\lambda} \frac{\exp \left(\frac{d}{\lambda}\right)}{1-\exp \left(\frac{2 d}{\lambda}\right)}
$$

where $C$ is a constant related to the spin-to-charge conversion efficiency of $\mathrm{Pt}$, the magnon injection-detection 
efficiencies, and the spin-mixing conductances at the interface between $\mathrm{MnPS}_{3}$ and $\mathrm{Pt}$.

Figure 3(b) shows the normalized nonlocal magnon resistance $\left(R_{\mathrm{NL}}^{*}\right)$ as a function of $d$ for the $16-\mathrm{nm} \mathrm{MnPS}_{3}$ device measured at $B=9 \mathrm{~T}$ and $T=2$ and $15 \mathrm{~K}$, respectively. $R_{\mathrm{NL}}^{*}$ is used to take into account of the length effect of the magnon detector, and it is calculated using the following formula: $R_{\mathrm{NL}}^{*}=R_{\mathrm{NL}}\left(1 / l_{P t_{-} D}\right)$, where $l_{P t_{-} D}$ is the length of the magnon detector. During the investigation of the magnon relaxation lengths, the spacings are purposely chosen to be longer than or equal to $2 \mu \mathrm{m}$, since there is a magnetic depletion region close to the Pt injector arising from local Joule heating induced spin Seebeck effect (Supplemental Material, Fig. S9) [43]. The spin signal probed from the local Pt injector is of opposite sign compared to the nonlocal Pt detector which probes the magnon transport across a spacing that is much bigger than the thickness of $\mathrm{MnPS}_{3}$ (Supplemental Material, Fig. S9 [39]).

To quantitatively determine the magnon relaxation length, the $\log \left(R_{\mathrm{NL}}^{*}\right)$ vs $d$ measured at various temperatures are plotted in Fig. 3(c). It is clear that the experimental results at various temperatures are all in good agreement with the exponential decay of magnon-dependent chemical potential expected theoretically [solid lines in Fig. 3(c)]. Based on the best-fitting results of the experimental data, the magnon relaxation lengths of the 16-nm $\mathrm{MnPS}_{3}$ are obtained to be $2.8 \pm 0.4 \mu \mathrm{m}$ at $T=2 \mathrm{~K}, 2.0 \pm 0.2 \mu \mathrm{m}$ at $T=10 \mathrm{~K}, 1.4 \pm 0.1 \mu \mathrm{m}$ at $T=15 \mathrm{~K}$, and $1.3 \pm 0.1 \mu \mathrm{m}$ at $T=20 \mathrm{~K}$, respectively. As shown in Fig. 3(d), the magnon relaxation length increases as temperature decreases, which could be attributed to the enhancement of the magnon lifetime at lower temperatures. This observation is consistent with previous reports of enhancement of magnon lifetimes at low temperatures in antiferromagnets $\mathrm{Cu}_{2} \mathrm{~V}_{2} \mathrm{O}_{7}$ and $\mathrm{MnF}_{2}$ investigated via spin Seebeckeffect measurements [50,51].

Next, the magnon transport and relaxation properties of quasi-2D $\mathrm{MnPS}_{3}$ are investigated as a function of the $\mathrm{MnPS}_{3}$ thickness. For this purpose, various magnon devices are fabricated on $\mathrm{MnPS}_{3}$ flakes with different thicknesses and the magnon relaxation length is obtained via the spacing dependence of the nonlocal spin signal. The exponential decay of magnon-dependent chemical potential has been observed on all the $\mathrm{MnPS}_{3}$ devices, which has been further confirmed on the $\mathrm{MnPS}_{3}$ devices with much larger spacings (Supplemental Material, Fig. S10 [39]). Figures 4(a) and 4(b) show the temperature dependence of the magnon relaxation lengths for the 8-nm $\mathrm{MnPS}_{3}$ device and the 27-nm $\mathrm{MnPS}_{3}$ device, respectively. The temperature dependences of the magnon relaxation lengths for these devices are similar to that measured on the 16-nm $\mathrm{MnPS}_{3}$ device [Fig. 3(d)].

Figure 4(c) shows the thickness dependence of the magnon relaxation lengths at $T=2,5$, and $10 \mathrm{~K}$, respectively. Clearly, an increase of the magnon relaxation length is (a)

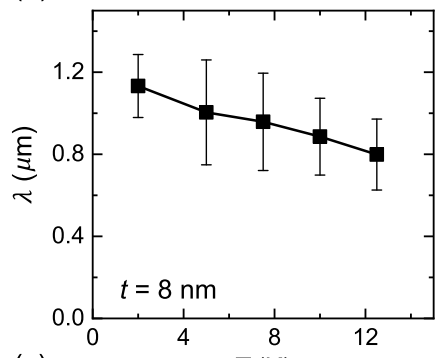

(b)

(c)

$T(\mathrm{~K})$
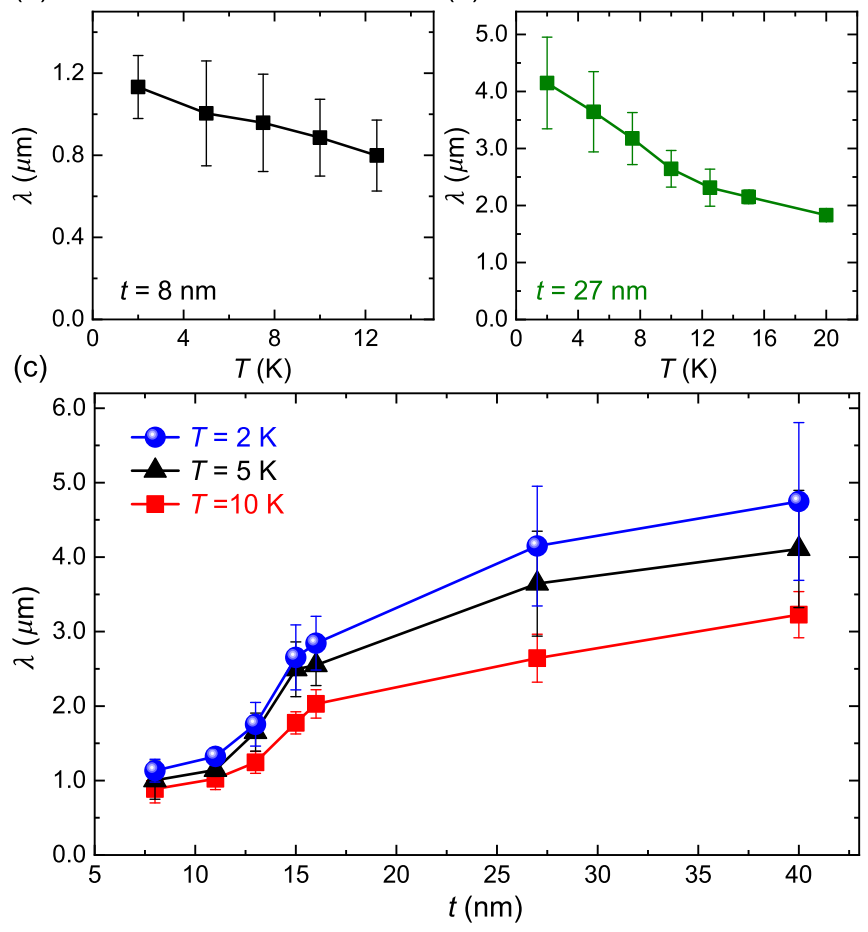

FIG. 4. Thickness dependence of magnon relaxation length of quasi-2D antiferromagnet $\mathrm{MnPS}_{3}$. (a),(b) The magnon relaxation length as a function of temperature for the 8- and 27-nm $\mathrm{MnPS}_{3}$ devices, respectively. (c) The $\mathrm{MnPS}_{3}$ thickness dependence of the magnon relaxation length obtained at $T=2,5$, and $10 \mathrm{~K}$, respectively.

observed as the $2 \mathrm{D} \mathrm{MnPS}_{3}$ thickness increases and it reaches $\sim 4.7 \mu \mathrm{m}$ for the 40-nm $\mathrm{MnPS}_{3}$ device. These magnon relaxation lengths for quasi-2D antiferromagnet $\mathrm{MnPS}_{3}$ are comparable to the values obtained in $3 \mathrm{D}$ ferromagnetic insulator YIG [30], which makes quasi-2D antiferromagnet $\mathrm{MnPS}_{3}$ a promising candidate for magnon spintronics applications. As $2 \mathrm{D} \mathrm{MnPS}_{3}$ thickness decreases, the magnon relaxation length decreases, i.e., a short magnon relaxation length of $\sim 1.1 \mu \mathrm{m}$ is obtained on the $8-\mathrm{nm} \mathrm{MnPS}_{3}$ device. These results indicate the presence of the enhanced magnon scattering for thinner $\mathrm{MnPS}_{3}$. One possible reason is the increase of surface-impurity-induced magnon scattering for thinner $\mathrm{MnPS}_{3}$ films, which gives rise to the strong suppression of the magnon lifetime. For devices fabricated on ultrathin $\mathrm{MnPS}_{3}$ flakes, no clear magnon transport signals have been observed yet (see Supplemental Material, Fig. S11 [39] for the results from two typical devices on 5-nm and 4-nm $\mathrm{MnPS}_{3}$ with $d=2 \mu \mathrm{m}$ ), which could be attributed to the presence of strong surface-impurityinduced magnon scattering and lower Néel temperature for thinner $\mathrm{MnPS}_{3}$, a Heisenberg antiferromagnet [46].

\section{CONCLUSIONS}

In conclusion, long-distance magnon transport over several micrometers has been demonstrated in the 
quasi-two-dimensional van der Waals antiferromagnet $\mathrm{MnPS}_{3}$. Systematical studies on the temperature and $\mathrm{MnPS}_{3}$ thickness dependences of the magnon relaxation lengths have been performed. As the $2 \mathrm{D} \mathrm{MnPS}_{3}$ thickness decreases, a shorter magnon diffusion length is observed, which could be attributed to the surface-impurity-induced magnon scattering. Our results demonstrate that van der Waals antiferromagnets provide a $2 \mathrm{D}$ platform for magnon spintronics and magnon spin computing [29]. Furthermore, these results could pave the way for the future investigation of novel magnon phenomena in van der Waals 2D magnets, including spin Nernst effect, magnon topological properties, quantum magnon Hall effect, etc. [33-35,37].

\section{ACKNOWLEDGMENTS}

We acknowledge the financial support from National Basic Research Programs of China (973 program Grants No. 2015CB921104, No. 2018YFA0305601, and No. 2015CB921102), National Natural Science Foundation of China (NSFC Grants No. 11574006, No. 11774007, No. 11534001, and No. U1832214) and the Strategic Priority Research Program of the Chinese Academy of Sciences (Grant No. XDB28020100).

[1] C. Gong, L. Li, Z. Li, H. Ji, A. Stern, Y. Xia, T. Cao, W. Bao, C. Wang, Y. Wang, Z. Q. Qiu, R. J. Cava, S. G. Louie, J. Xia, and X. Zhang, Discovery of Intrinsic Ferromagnetism in Two-Dimensional van der Waals Crystals, Nature (London) 546, 265 (2017).

[2] B. Huang, G. Clark, E. Navarro-Moratalla, D. R. Klein, R. Cheng, K. L. Seyler, D. Zhong, E. Schmidgall, M. A. McGuire, D. H. Cobden, W. Yao, D. Xiao, P. JarilloHerrero, and $\mathrm{X}$. Xu, Layer-Dependent Ferromagnetism in a van der Waals Crystal down to the Monolayer Limit, Nature (London) 546, 270 (2017).

[3] W. Xing, Y. Chen, P. M. Odenthal, X. Zhang, W. Yuan, T. Su, Q. Song, T. Wang, J. Zhong, S. Jia, X. C. Xie, Y. Li, and W. Han, Electric Field Effect in Multilayer $\mathrm{Cr}_{2} \mathrm{Ge}_{2} \mathrm{Te}_{6}$ : A Ferromagnetic 2D Material, 2D Mater. 4, 024009 (2017).

[4] J.-U. Lee, S. Lee, J. H. Ryoo, S. Kang, T. Y. Kim, P. Kim, C.-H. Park, J.-G. Park, and H. Cheong, Ising-Type Magnetic Ordering in Atomically Thin $\mathrm{FePS}_{3}$, Nano Lett. 16, 7433 (2016).

[5] X. Wang, K. Du, Y. Y. F. Liu, P. Hu, J. Zhang, Q. Zhang, M. H. S. Owen, X. Lu, C. K. Gan, P. Sengupta, C. Kloc, and Q. Xiong, Raman Spectroscopy of Atomically Thin TwoDimensional Magnetic Iron Phosphorus Trisulfide $\left(\mathrm{FePS}_{3}\right)$ Crystals, 2D Mater. 3, 031009 (2016).

[6] N. Samarth, Condensed-Matter Physics: Magnetism in Flatland, Nature (London) 546, 216 (2017).

[7] S. Liu, X. Yuan, Y. Zou, Y. Sheng, C. Huang, E. Zhang, J. Ling, Y. Liu, W. Wang, C. Zhang, J. Zou, K. Wang, and F. Xiu, Wafer-Scale Two-Dimensional Ferromagnetic $\mathrm{Fe}_{3} \mathrm{GeTe}_{2}$ Thin Films Grown by Molecular Beam Epitaxy, npj 2D Mater. Appl. 1, 30 (2017).
[8] M. Bonilla, S. Kolekar, Y. Ma, H. C. Diaz, V. Kalappattil, R. Das, T. Eggers, H. R. Gutierrez, M.-H. Phan, and M. Batzill, Strong Room-Temperature Ferromagnetism in $\mathrm{VSe}_{2}$ Monolayers on van der Waals Substrates, Nat. Nanotechnol. 13, 289 (2018).

[9] D. J. O’Hara, T. Zhu, A. H. Trout, A. S. Ahmed, Y. (Kelly) Luo, C. H. Lee, M. R. Brenner, S. Rajan, J. A. Gupta, D. W. McComb, and R. K. Kawakami, Room Temperature Intrinsic Ferromagnetism in Epitaxial Manganese Selenide Films in the Monolayer Limit, Nano Lett. 18, 3125 (2018).

[10] Y. Tian, M. J. Gray, H. Ji, R. J. Cava, and K. S. Burch, Magneto-Elastic Coupling in a Potential Ferromagnetic 2D Atomic Crystal, 2D Mater. 3, 025035 (2016).

[11] D. Weber, L. M. Schoop, V. Duppel, J. M. Lippmann, J. Nuss, and B. V. Lotsch, Magnetic Properties of Restacked 2 D Spin 1/2 Honeycomb $\mathrm{RuCl}_{3}$ Nanosheets, Nano Lett. 16, 3578 (2016).

[12] B. Zhou, Y. Wang, G. B. Osterhoudt, P. Lampen-Kelley, D. Mandrus, R. He, K. S. Burch, and E. A. Henriksen, Possible Structural Transformation and Enhanced Magnetic Fluctuations in Exfoliated $\alpha$ - $\mathrm{RuCl}_{3}$, J. Phys. Chem. Solids, DOI: 10.1016/j.jpcs.2018.01.026.

[13] M. Ziatdinov, A. Banerjee, A. Maksov, T. Berlijn, W. Zhou, H. B. Cao, J. Q. Yan, C. A. Bridges, D. G. Mandrus, S. E. Nagler, A. P. Baddorf, and S. V. Kalinin, Atomic-Scale Observation of Structural and Electronic Orders in the Layered Compound $\alpha-\mathrm{RuCl}_{3}$, Nat. Commun. 7, 13774 (2016).

[14] S. Mashhadi, D. Weber, L. M. Schoop, A. Schulz, B. V. Lotsch, M. Burghard, and K. Kern, Electrical Transport Signature of the Magnetic Fluctuation-Structure Relation in $\alpha-\mathrm{RuCl}_{3}$ Nanoflakes, Nano Lett. 18, 3203 (2018).

[15] K. S. Burch, D. Mandrus, and J.-G. Park, Magnetism in Two-Dimensional van der Waals Materials, Nature (London) 563, 47 (2018).

[16] N. D. Mermin and H. Wagner, Absence of Ferromagnetism or Antiferromagnetism in One- or Two-Dimensional Isotropic Heisenberg Models, Phys. Rev. Lett. 17, 1133 (1966).

[17] D. R. Klein, D. MacNeill, J. L. Lado, D. Soriano, E. Navarro-Moratalla, K. Watanabe, T. Taniguchi, S. Manni, P. Canfield, J. Fernández-Rossier, and P. Jarillo-Herrero, Probing Magnetism in 2D van der Waals Crystalline Insulators via Electron Tunneling, Science 360, 1218 (2018).

[18] T. Song, X. Cai, M. W.-Y. Tu, X. Zhang, B. Huang, N. P. Wilson, K. L. Seyler, L. Zhu, T. Taniguchi, K. Watanabe, M. A. McGuire, D. H. Cobden, D. Xiao, W. Yao, and X. Xu, Giant Tunneling Magnetoresistance in Spin-Filter van der Waals Heterostructures, Science 360, 1214 (2018).

[19] Z. Wang, I. Gutiérrez-Lezama, N. Ubrig, M. Kroner, T. Taniguchi, K. Watanabe, A. Imamoğlu, E. Giannini, and A. F. Morpurgo, Very Large Tunneling Magnetoresistance in Layered Magnetic Semiconductor $\mathrm{CrI}_{3}$, Nat. Commun. 9 , 2516 (2018).

[20] S. S. P. Parkin, C. Kaiser, A. Panchula, P. M. Rice, B. Hughes, M. Samant, and S.-H. Yang, Giant Tunnelling Magnetoresistance at Room Temperature with $\mathrm{MgO}$ (100) Tunnel Barriers, Nat. Mater. 3, 862 (2004).

[21] S. Yuasa, T. Nagahama, A. Fukushima, Y. Suzuki, and K. Ando, Giant Room-Temperature Magnetoresistance in 
Single-Crystal Fe/MgO/Fe Magnetic Tunnel Junctions, Nat. Mater. 3, 868 (2004).

[22] D. Ghazaryan, M. T. Greenaway, Z. Wang, V. H. GuarochicoMoreira, I. J. Vera-Marun, J. Yin, Y. Liao, S. V. Morozov, O. Kristanovski, A. I. Lichtenstein, M. I. Katsnelson, F. Withers, A. Mishchenko, L. Eaves, A. K. Geim, K. S. Novoselov, and A. Misra, Magnon-Assisted Tunnelling in van der Waals Heterostructures Based on $\mathrm{CrBr}_{3}$, Nat. Electron. 1, 344 (2018).

[23] S. Jiang, J. Shan, and K. F. Mak, Electric-Field Switching of Two-Dimensional van der Waals Magnets, Nat. Mater. 17, 406 (2018).

[24] B. Huang, G. Clark, D. R. Klein, D. MacNeill, E. NavarroMoratalla, K. L. Seyler, N. Wilson, M. A. McGuire, D. H. Cobden, D. Xiao, W. Yao, P. Jarillo-Herrero, and X. Xu, Electrical Control of 2D Magnetism in Bilayer $\mathrm{CrI}_{3}$, Nat. Nanotechnol. 13, 544 (2018).

[25] Y. Deng, Y. Yu, Y. Song, J. Zhang, N. Z. Wang, Y. Z. Wu, J. Zhu, J. Wang, X. H. Chen, and Y. Zhang, Gate-Tunable Room-Temperature Ferromagnetism in Two-Dimensional $\mathrm{Fe}_{3} \mathrm{GeTe}_{2}$, Nature (London) 563, 94 (2018).

[26] Z. Wang, T. Zhang, M. Ding, B. Dong, Y. Li, M. Chen, X. Li, J. Huang, H. Wang, X. Zhao, Y. Li, D. Li, C. Jia, L. Sun, H. Guo, Y. Ye, D. Sun, Y. Chen, T. Yang, J. Zhang et al., Electric-Field Control of Magnetism in a Few-Layered van der Waals Ferromagnetic Semiconductor, Nat. Nanotechnol. 13, 554 (2018).

[27] T. Dietl, A Ten-Year Perspective on Dilute Magnetic Semiconductors and Oxides, Nat. Mater. 9, 965 (2010).

[28] T. Dietl and H. Ohno, Dilute Ferromagnetic Semiconductors: Physics and Spintronic Structures, Rev. Mod. Phys. 86, 187 (2014).

[29] A. V. Chumak, V. I. Vasyuchka, A. A. Serga, and B. Hillebrands, Magnon Spintronics, Nat. Phys. 11, 453 (2015).

[30] L. J. Cornelissen, J. Liu, R. A. Duine, J. B. Youssef, and B. J. van Wees, Long-Distance Transport of Magnon Spin Information in a Magnetic Insulator at Room Temperature, Nat. Phys. 11, 1022 (2015).

[31] W. Yuan, Q. Zhu, T. Su, Y. Yao, W. Xing, Y. Chen, Y. Ma, X. Lin, J. Shi, R. Shindou, X. C. Xie, and W. Han, Experimental Signatures of Spin Superfluid Ground State in Canted Antiferromagnet $\mathrm{Cr}_{2} \mathrm{O}_{3}$ via Nonlocal Spin Transport, Sci. Adv. 4, eaat1098 (2018).

[32] R. Lebrun, A. Ross, S. A. Bender, A. Qaiumzadeh, L. Baldrati, J. Cramer, A. Brataas, R. A. Duine, and M. Kläui, Tunable Long-Distance Spin Transport in a Crystalline Antiferromagnetic Iron Oxide, Nature (London) 561, 222 (2018).

[33] R. Cheng, S. Okamoto, and D. Xiao, Spin Nernst Effect of Magnons in Collinear Antiferromagnets, Phys. Rev. Lett. 117, 217202 (2016).

[34] K. Nakata, S. K. Kim, J. Klinovaja, and D. Loss, Magnonic Topological Insulators in Antiferromagnets, Phys. Rev. B 96, 224414 (2017).

[35] Y. Su, X. S. Wang, and X. R. Wang, Magnonic Weyl Semimetal and Chiral Anomaly in Pyrochlore Ferromagnets, Phys. Rev. B 95, 224403 (2017).

[36] J. Lan, W. Yu, and J. Xiao, Antiferromagnetic Domain Wall as Spin Wave Polarizer and Retarder, Nat. Commun. 8, 178 (2017).
[37] B. Xu, T. Ohtsuki, and R. Shindou, Integer Quantum Magnon Hall Plateau-Plateau Transition in a Spin-Ice Model, Phys. Rev. B 94, 220403 (2016).

[38] K. Okuda, K. Kurosawa, S. Saito, M. Honda, Z. Yu, and M. Date, Magnetic Properties of Layered Compound $\mathrm{MnPS}_{3}$, J. Phys. Soc. Jpn. 55, 4456 (1986).

[39] See Supplemental Material at http://link.aps.org/ supplemental/10.1103/PhysRevX.9.011026 for the determination of Néel temperature, atomic force microscopy measurement, Raman characterization, the first and second harmonic nonlocal results, the current dependence of the second harmonic voltage, the insulating properties of quasi2D MnPS3, nonlocal measurements on control devices, local vs nonlocal measurements, spacing dependence on extra devices with much larger sapcings, and magnon transport in ultrathin 2D MnPS3 devices.

[40] S. T. B. Goennenwein, R. Schlitz, M. Pernpeintner, K. Ganzhorn, M. Althammer, R. Gross, and H. Huebl, NonLocal Magnetoresistance in YIG/Pt Nanostructures, Appl. Phys. Lett. 107, 172405 (2015).

[41] L. J. Cornelissen, K. J. H. Peters, G. E. W. Bauer, R. A. Duine, and B. J. van Wees, Magnon Spin Transport Driven by the Magnon Chemical Potential in a Magnetic Insulator, Phys. Rev. B 94, 014412 (2016).

[42] L. J. Cornelissen, J. Shan, and B. J. van Wees, Temperature Dependence of the Magnon Spin Diffusion Length and Magnon Spin Conductivity in the Magnetic Insulator Yttrium Iron Garnet, Phys. Rev. B 94, 180402 (2016).

[43] J. Shan, L. J. Cornelissen, J. Liu, J. B. Youssef, L. Liang, and B. J. van Wees, Criteria for Accurate Determination of the Magnon Relaxation Length from the Nonlocal Spin Seebeck Effect, Phys. Rev. B 96, 184427 (2017).

[44] B. L. Giles, Z. Yang, J. S. Jamison, and R. C. Myers, Long-Range Pure Magnon Spin Diffusion Observed in a Nonlocal Spin-Seebeck Geometry, Phys. Rev. B 92, 224415 (2015).

[45] C. Safranski, I. Barsukov, H. K. Lee, T. Schneider, A. A. Jara, A. Smith, H. Chang, K. Lenz, J. Lindner, Y. Tserkovnyak, M. Wu, and I. N. Krivorotov, Spin Caloritronic Nano-oscillator, Nat. Commun. 8, 117 (2017).

[46] P. A. Joy and S. Vasudevan, Magnetism in the Layered Transition-Metal Thiophosphates $\mathrm{MPS}_{3}(M=\mathrm{Mn}, \mathrm{Fe}$, and Ni), Phys. Rev. B 46, 5425 (1992).

[47] T. Jungwirth, X. Marti, P. Wadley, and J. Wunderlich, Antiferromagnetic Spintronics, Nat. Nanotechnol. 11, 231 (2016).

[48] V. Baltz, A. Manchon, M. Tsoi, T. Moriyama, T. Ono, and Y. Tserkovnyak, Antiferromagnetic Spintronics, Rev. Mod. Phys. 90, 015005 (2018).

[49] T. Valet and A. Fert, Theory of the Perpendicular Magnetoresistance in Magnetic Multilayers, Phys. Rev. B 48, 7099 (1993).

[50] Y. Shiomi, R. Takashima, D. Okuyama, G. Gitgeatpong, P. Piyawongwatthana, K. Matan, T. J. Sato, and E. Saitoh, Spin Seebeck Effect in the Polar Antiferromagnet $\alpha-\mathrm{Cu}_{2} \mathrm{~V}_{2} \mathrm{O}_{7}$, Phys. Rev. B 96, 180414 (2017).

[51] S. M. Wu, W. Zhang, A. Kc, P. Borisov, J. E. Pearson, J. S. Jiang, D. Lederman, A. Hoffmann, and A. Bhattacharya, Antiferromagnetic Spin Seebeck Effect, Phys. Rev. Lett. 116, 097204 (2016). 